\title{
An Integrated Account of Rosen's Relational Biology and Peirce's Semiosis. Part I: Components and Signs, Final Cause and Interpretation
}

\author{
Federico Vega ${ }^{1}$ (D) \\ Received: 13 May 2021 / Accepted: 11 July 2021/Published online: 15 September 2021 \\ (C) The Author(s) 2021
}

\begin{abstract}
Robert Rosen's relational biology and biosemiotics share the claim that life cannot be explained by the laws that apply to the inanimate world alone. In this paper, an integrated account of Rosen's relational biology and Peirce's semiosis is proposed. The ultimate goal is to contribute to the construction of a unified framework for the definition and study of life. The relational concepts of component and mapping, and the semiotic concepts of sign and triadic relation are discussed and compared, and a representation of semiotic relations with mappings is proposed. The role of the final cause in two theories that account for what differentiates living beings, natural selection and relational biology, is analyzed. Then the presence of the final cause in Peirce's semiosis is discussed and, with it, the similarities and differences between the theories of Rosen and Peirce are deepened. Then, a definition of a semiotic relation in an organism is proposed, and Short's definition of interpretation is applied. Finally, a method to identify and analyze semiotic actions in an organism is proposed.
\end{abstract}

Keywords Relational biology $\cdot$ Semiosis $\cdot$ Sign $\cdot$ Component $\cdot$ Final cause $\cdot$ Interpretation.

\section{Introduction}

In relational biology, organisms are analyzed as systems made up of components that are related to each other, producing system closure to efficient cause. It is closure that defines the living being, turns the parts of a natural system into components that perform functions and gives the relations between natural entities its own reality.

On the other hand, biosemiotics maintains that it is the use of signs that characterizes the living being. Unlike dynamic actions, semiotic actions correspond to triadic relations

Federico Vega

federico.vega@telefonica.net

1 Department of Logic, History and Philosophy of Science, Universidad Nacional de Educación a Distancia, Madrid, Spain 
between the subjects that make up the semiotic relation (sign, object and interpretant) that cannot be reduced to dyadic relations.

Moreover, as has been explained by various authors (e.g. Hoffmeyer ${ }^{1}$ ), the concept of a sign is a relational concept. Furthermore, it is argued below that semiosis is doubly relational: a semiotic relation is a local triadic relation among three subjects, and the semiotic action participates in the systemic closure that defines the organism.

In this paper, an integrated account of Rosen's relational biology and Peirce's semiosis is proposed.

Section on "Components, Signs, Mappings and Triadic Relations" begins with the presentation of Rosen's concepts of natural law, model, component and mapping and his analysis of Aristotelian causes. Next, considering Peirce's definition of a sign, the possibility of representing semiotic relations with mappings is discussed and a proposal is made. Finally, it is argued that, although the action of a component and the interpretation of a sign can be reduced to dyadic relations, the semiotic relation, which includes the interpretation of the sign and determination of the interpretant, cannot.

In section "Final Cause", the similarities and differences between the theories of Rosen and Peirce previously pointed out are deepened. First, natural selection is presented as an instrument to explain the reintroduction of the final cause in science and for the inspirational power it had for Peirce. Second, it is discussed Rosen's proposal for the final cause, based on the concept of closure. Both natural selection and relational biology are applied to living beings and they complement each other: one explains how new organisms appear through the reproduction of existing ones and the other characterizes what differentiates living beings from inanimate matter. Finally, the role of the final cause in Peirce's semiosis is studied. All three theories require the introduction of new explananda (natural selection, closure to efficient cause and Peirce's sign) that are part of three corresponding new explanans.

The similarities and differences established in the previous sections between the theories of Rosen and Peirce, as well as the analysis of the role that the final cause plays in them constitute an adequate tool to discuss the existence of signs that realize and are interpreted inside organisms. Section "Signs in an Organism" begins with the analysis of Short's definition of interpretation. However, Short argues that only in the animal realm can one speak of sign, interpretation and meaning. To overcome this limitation and extend the use of these concepts to the level of the simplest organism, the conceptualization of "intentionality" in biosemiotics is reviewed. As a result, a semiotic relation in an organism is defined. Finally, the concepts of purpose and interpretation are discussed, and it is analyzed who is the agent that performs the interpretation in an organism.

Finally, the section "A Method of Analysis", presents the steps to follow to identify the presence of signs in an organism.

\section{Components, Signs, Mappings and Triadic Relations}

\section{Components, Mappings and Aristotelian Causes}

According to Rosen (2012):

\footnotetext{
${ }^{1}$ (...) the essence of the sign is its formal relational character of evoking an awareness of something which it is not itself - and thereby implying the full Peircean triad of sign, object and interpretant (Hoffmeyer, 2008, p. 18).
} 
A natural system is essentially a bundle of linked qualities, or observables, coded or named by the specific percepts which they generate, and by the relations which the mind creates to organize them. (...) A formal system, on the other hand, is entirely a creation of the mind, possessing no properties beyond those which enter its definition and their implications. (...) We are going to force the name of a percept to be also the name of a formal entity; we are going to force the name of a linkage between percepts to also be the name of a relation between mathematical entities; and most particularly, we are going to force the various temporal relations characteristic of causality in the natural world to be synonymous with the inferential structure which allows us to draw conclusions from premises in the mathematical world (pp. 71-72).

As explained in Rosen (1991), the concept of natural law is based on the assertion that there exists, and can be established, a relation between two different modes of implication: the causality of natural systems and the inferential implication of formal systems. This relation is established through a model. Consequently, a natural law would be the congruence between the causality of a natural system and the inferential implication of a formal system that is a model of the natural system.

The formal systems proposed by Rosen to model natural systems are relational systems. Consider a separable part of a natural system. The difference in the behavior of the complete system, compared to the case in which a part has been separated, defines the function of the separated part. A component is a part of the system that can be assigned a function and is the basic unit of the organization of the system. A component can be represented as a mapping between two sets, f: $\mathbf{A} \rightarrow \mathbf{B}$. This implication can be expanded as $\mathbf{f} \longrightarrow(\mathbf{a} \longrightarrow \mathbf{f}(\mathbf{a}))$, for every $\mathbf{a} \in \mathbf{A}$, with $\mathbf{f}(\mathbf{a})=$ $\mathbf{b} \in \mathbf{B}$, and represented graphically as indicated in Fig. 1A and B, where hollow-headed arrows represent the flow from $\mathbf{a}$ to $\mathbf{b}$, and solid-headed arrows symbolise the effect of the component. In a component, $\mathbf{f}$ entails that $\mathbf{a}$ entails $\mathbf{b}$.

In Fig. 1B, b represents a quality of the natural system. To explain the realization of this quality, to answer the question "why b?" Rosen relies on the four Aristotelian causes: material, efficient, formal and final.

Following Rosen, we find two of them represented in the mapping. First, the qualities represented by $\mathbf{A}$ are the material causes of the qualities represented by $\mathbf{B}$. In short, we will say that $\mathbf{A}$ is the material cause of $\mathbf{B}$, and specifically, each element of $\mathbf{A}$ is the material cause of an element of $\mathbf{B}$. Second, $\mathbf{f}$ corresponds to the efficient cause of $\mathbf{B}$ (of each element of $\mathbf{B}$ ).

On the other hand, according to Louie, we can consider that the formal cause is represented by the mapping structure shown in Fig. 2:

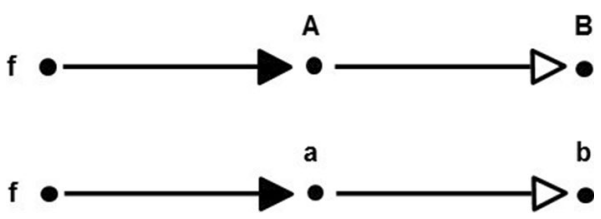

Fig. 1 A representation of a component, $\mathbf{B}$ Component action on a specific input 


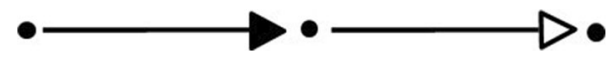

Fig. 2 Representation of the formal cause, according to Louie

The mapping's form, or its statement of essence, is the structure of the mapping itself as a morphism (...) the formal cause of the mapping is thus the ordered pair of arrows (Louie 2009, p. 111).

Finally, Louie also proposes a representation for the final cause (See Fig. 3):

The Greek term $\tau \varepsilon ́ \lambda o \zeta$ (télos, translated into finis in Latin), meaning 'end' or 'purpose', covers two meanings: the end considered as the object entailed (i.e., b itself), or the end considered as the entailment of the object (i.e., the production of b). In both cases, the final cause may be identified as b, whence also the hollowheaded arrow that terminates on the output (Louie 2009, p. 113).

However, when we ask, "why b?" the answer cannot be "because b".

In section "Final Cause", the final cause in relational biology is analyzed and a proposal is made on its representation.

\section{Signs and Mappings}

It is worth considering now whether it would be possible to represent semiotic relations with mappings, and if this representation could be useful in the analysis of semiotic actions.

To answer this question, it is appropriate to start with Peirce:

A sign, or representamen (...) addresses somebody, that is, creates in the mind of that person an equivalent sign, or perhaps a more developed sign. That sign which it creates I call the interpretant of the first sign. The sign stands for something, its object. It stands for that object, not in all respects, but in reference to a sort of idea, which I have sometimes called the ground of the representamen.

A Sign, or Representamen, is a First which stands in such a genuine triadic relation to a Second, called its Object, as to be capable of determining a Third, called its Interpretant (Peirce as quoted in Favareau (2009, p. 122)).

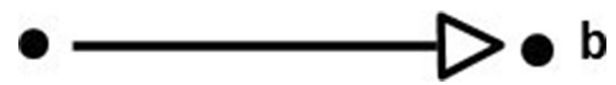

Fig. 3 Representation of the final cause, according to Louie 


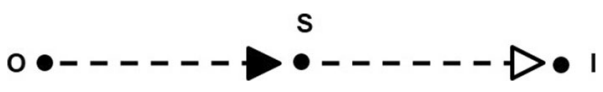

Fig. 4 Determination of the interpretant

I define a sign as anything which is so determined by something else, called its Object, and so determines an effect upon a person, which effect I call its interpretant, that the latter is thereby mediately determined by the former" (Piece 1998, p. 478).

Two comments deserve to be extracted from these quotations. First, an object determines a sign insofar as not just any subject can stand for that object. The object determines a sign among the subjects that present some aspects that can stand for it. The same object can determine several signs that have different characteristics that can stand for it, which would give rise to several semiotic relations.

Second, the aspects of the sign that are suitable to stand for the object determine the idea that is created. Then, the determination of the sign determines the interpretant. In this sense, the object entails that the sign entails the interpretant. Consequently, the determination of the interpretant can be represented as shown in Fig. 4, where $\mathbf{I}, \mathbf{S}$ and $\mathbf{O}$ stand respectively for the interpretant, the sign and the object.

Dashed lines are used in the mapping that represents the determination of the interpretant to indicate that it is a mapping that does not correspond to a component of an organism.

On the other hand, as explained in Short's definition of interpretation (Short, 2007) discussed in the section named "Signs in an Organism", the interpretation responds to the purpose of an agent for which the interpretant leads from the representamen to the object. For the agent, the interpretant entails that (excepting failures) the sign entails the object. Accordingly, the interpretation of the sign (the semiotic action) can be represented as shown in Fig. 5.

Continuous lines are used in the mapping that represents the interpretation of the sign since, as argued in the aforementioned section, if it is a sign that belongs to an organism, its interpretation corresponds to the action of one of its components.

Putting the above considerations together, a semiotic relation can be represented with two mappings as shown in Fig. 6, corresponding to its realization and interpretation.

Neither of the two mappings by itself fully represent the semiotic relation. What represents the semiotic relation is the union of both.

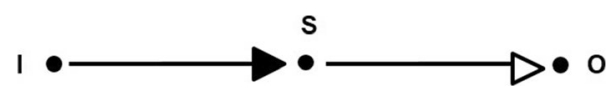

Fig. 5 Interpretation of the sign 


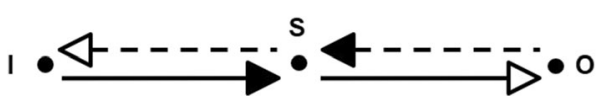

Fig. 6 A semiotic relation

\section{Dyadic and Triadic Relations}

A component of a relational system could be associated, in principle, with the triadic relation (f, $\mathbf{a}, \mathbf{b}){ }^{2}$ However, as discussed above, $\mathbf{f}$ entails than a entails $\mathbf{b}$, and thus the component action can be reduced to nested dyadic relations (f, $(\mathbf{a}, \mathbf{b}))$. Conversely, in Peirce's semiosis, the concepts of interpretation and meaning are inseparable from the relation that is established between sign, object, and interpretant; a triadic relation that cannot be reduced to dyadic relations.

As argued below, in a natural system, the interpretation of a sign corresponds to the action of a component of an organism. Accordingly, it could be explained as the triadic relation (interpretant, sign, object) that can be reduced to the nested dyadic relations (interpretant, (sign, object)). However, the definition of the sign also requires the presence of the formal relation that corresponds to the determination of the interpretant. The recursive relations established between interpretant, sign and object that are represented with the two mappings in Fig. 6 can be associated to a triadic relation that cannot be reduced to dyadic relations. Although the interpretation of the sign, considered separately, can be reduced to dyadic relations, the semiotic relation, which includes the interpretation and determination of the interpretant, cannot.

Finally, it is important to note that, whenever the interpretant of a sign is associated with the efficient cause of a component, it would be more correct to associate it with the union of the efficient and formal causes. As indicated in footnote 2, in a natural system, efficient and formal causes are not separable. To simplify the discussion, references to the formal cause are omitted.

\section{Final Cause}

The ultimate and distinctive goal of biology is the definition of life, the explanation of what differentiates an organism from an inanimate system. According to Rosen, the explanation of life requires a science broader than that which explains the inanimate world.

In relational biology, an organism is a set of interrelated components. But the organism is more than the sum of all its parts. According to Rosen, life cannot be explained with the laws of physics alone; a complete description of an organism cannot be the description of a mechanism. ${ }^{3}$ An organism is a complex system that can have mechanistic (and non-mechanistic) models, but the limit of which is not a mechanism.

\footnotetext{
$\overline{2}$ In principle, the tetradic relation (f, for, $\mathbf{a}, \mathbf{b}$ ), where $\mathbf{f}$, for, $\mathbf{a}$ and $\mathbf{b}$ stand respectively for the efficient and formal causes, the input and the output, should be considered. However, it must be borne in mind that, in a natural system, efficient and formal causes are not separable; they lead together from the input a to the output b. For simplicity, references to the formal cause are omitted.

3 Rosen's concept of mechanism is intrinsically linked to the definition of law of nature inherited from Newton. For an analysis of this point, see Vega (2018).
} 
In each component of the system, obtaining the output from the input can be explained by its material, efficient and formal causes; it can, in principle, be explained with the laws of physics alone; it admits, in principle, a mechanistic explanation. However, for the explanation of life, these three Aristotelian causes are not enough, the laws of physics are not sufficient, mechanistic explanations are not enough. Explanation of life requires the introduction of the final cause.

In this section, the role of the final cause in natural selection, relational biology and Peirce's semiosis is discussed.

\section{Final Cause in Natural Selection}

As Short (2002) explains, Aristotle conceives final causes as types of outcomes. While an efficient cause always leads to a particular outcome, a final cause is a type of outcome that can be realized in different ways and, furthermore, the actualization of a particular of that outcome type may fail. However, not all types of outcomes are final causes; a type of outcome is a final cause only if it explains why there tend to be instances of that type.

The reintroduction of the classical concept of final cause in modern science is necessary because there are questions that efficient causes cannot answer. Short argues that there are explananda that are not mechanistically explainable:

My point is that a pattern discernible in events is an aspect distinct from the sum of the individual events that compose the pattern. Thus, the pattern requires an explanation - unless, indeed, it is coincidental - distinct from the logical union of the explanations of the individual events $(2002,331){ }^{4}$

Furthermore, to explain an explanandum of this type, the final cause must also provide a different form of explanation; a way in which the explanandum figures in the explanans. The tendency for outcomes of a certain type to occur is explained by what that type is. The aforementioned work analyzes what natural selection explains and how it does it, and it is stated that Darwin's use of the concept of final cause coincides with that of Aristotle. The development of adaptations, and the evolution and diversity of species constitutes the distinctive explanandum of natural selection. An explanandum for which the laws of physics ${ }^{5}$ are not sufficient, as it cannot be explained just by tracing mechanical processes.

Moreover, the explanandum figures in the explanans: the consequences that explain the existence of adaptive traits are the consequences that these traits have, and the consequences precede the effect they explain. However, the final causation is not a "backwards causation" because the type of outcome is not a particular outcome: there is no particular outcome that has influence on a particular income that precedes it in time.

\footnotetext{
4 See the correspondence between this statement and the tenets of relational biology.

5 When referring to the laws of physics in this work, this expression must be understood in a broad sense from two perspectives. First, it is about the laws of nature that come from Newton but that include recent contributions to this discipline, even those that incorporate different conceptions from the traditional ones, such as quantum mechanics. Second, it also includes the laws of science that can be derived, in principle, from physics, such as chemistry.
} 
According to natural selection, evolution is shaped by types of outcome, and the outcomes that are realized are explained by the types they exemplify. The types of outcomes are, therefore, final causes, and they are explanatory because outcomes are selected for being of those types.

In natural selection, the selected type is different from the selected genotype. The type selected is part of the explanation for the selection of a particular genotype. This genotype is selected for exemplifying that type more efficiently than the other genotypes available at the time the selection is made.

\section{Final Cause in Relational Biology}

Rosen (1991) posits that relying on natural selection to explain final causes is a false solution to the problem. He recalls that the way in which the parts of a system interact between themselves and contribute to the overall functioning of the system constitutes its physiology, whereas the way the system was formed from different parts constitutes its origin. The modes of implication involved in evolutionary processes and those involved in physiological processes are different.

Both natural selection and relational biology are applied to living beings; the first to explain how new organisms appear through the reproduction of existing ones, the second to characterize what differentiates living beings from inanimate matter. In Darwin's account, the mechanisms of inheritance introduce in natural science a new explanatory principle, natural selection, which account for the selection of characteristics for types of effects that lead to reproductive success. According to Rosen, the definition of life also requires the introduction of a new explanatory principle: closure to efficient cause, explained below. In relational biology, the explanation of the final cause and the definition of an organism is based on the concept of closure to efficient cause, and this closure explains what the system is like at the present time, without referring to its origin.

The analysis of Rosen's concept of final cause should start from highlighting something that differentiates it from other Aristotelian causes: clearly, the material, efficient and formal causes of the effect produced by a component in the system to which it belongs imply said effect; conversely, the final cause must be defined in terms of something implied by the effect.

According to Rosen, in a certain sense, the effect entails the component:

(...) it is perfectly respectable to talk about a category of final causation and to identify a component as the effect of its final cause, its function in the diagram (...) a component is entailed by its function (...) we gain (...) another independent mode of entailment, it is a mode that entails a mapping (...) I shall call it functional entailment $^{6}$, distinct from the inner and outer entailments (...) (Rosen 1991, p. 134). As Hoffmeyer points out:

\footnotetext{
6 Louie (2009) calls this type of entailment "immanent entailment", and uses "functional entailment" for the case in which the output of a mapping is another mapping.
} 
Rosen defended a return to Aristotle's broader understanding of causality, but subjected the term "functional entailment" in place of "final cause", using functional in its biological sense (Hoffmeyer, 2013, footnote 29, p. 166).

Then, an answer to the question "why b?" can be "because b performs a function that entails the component of which $\mathbf{b}$ is an output".

The explanatory principle proposed by Rosen is the closure to efficient cause. The concept of closure ${ }^{7}$ in general terms designates a feature of biological systems by virtue of which their constitutive components and operations depend on each other for their production and maintenance and also collectively contribute to determine the conditions under which the system itself can exist.

Varela (1979) proposed the "Closure Thesis", according to which every autonomous system is an operationally closed system. For Varela, closure is understood as closure of processes. Starting from any point in a component production network, the chained processes convert inputs into outputs and back to the starting component. An autopoietic system (Maturana and Varela 1973, Varela 1979) is an operationally closed system and therefore a system capable of self-maintaining, reproducing and generating diversity. An autopoietic system is, for them, a living being. Following Maturana and Varela, the question "why b?" in Fig. 1B can be answered "because b contributes to the operational closure of the system". Thus, in an organism, the final cause of a process is its contribution to the operational closure of the system and thus its contribution to self-maintenance and reproduction of the system, and to the generation of diversity.

In Rosen's (1991) account, a material system is an organism if and only if it is closed to efficient cause. A system is closed to efficient cause if its components have efficient causes generated within the system, and effects that contribute to the production of other efficient causes. Furthermore, the functions of the components contribute to the production of other functions, and to the organization and maintenance of the system. In a system subjected to a closure to efficient cause, a component that contributes to that closure exerts a function, and performing a function is equivalent to contributing to closure. Closure to efficient cause generates a set of functions: functionality is an emergent property of closure. The question "why b? " can be answered "because $\mathbf{b}$ contributes to closure to efficient cause of the system to which the component is part", or, equivalently, "because b performs a function in the system". Therefore, the final cause of a component of an organism is the biological function that the component performs in that organism, or, equivalently, the contribution of the biological function to the closure of the system.

When explaining the (M, R)-systems, Rosen (1991) points out that it is closure to efficient cause that solves the problems of metabolism, repair and replication of the system. In an organism, the final cause of a component is its contribution to the self-maintenance of the (metabolism, self-repair and organizational invariance of the) system.

Uniting Rosen's concepts of functional entailment (final cause) and closure, the question "why b?" can be answered "because b performs a function that, through closure to efficient cause, entails the component of which $\mathbf{b}$ is an output".

\footnotetext{
7 The notion of organizational closure was introduced in 1967 by Piaget (1967).
} 
Consequently, it is proposed to represent the final cause as shown in Fig. 7. In a relational model, the representation of the final cause of a component is not found within the mapping that represents the component, but in the relation of said mapping with those that represent other components with which it contributes to the closure of the system ${ }^{8}$.

Besides, it is worth asking not only for the final cause of a component, but also for the final cause of an organism as such. ("Why is there a system closed to efficient cause?"). Closure to efficient cause is a type of organization that explains why there tend to be systems that exemplify that type. The realization of a closure to efficient cause is explained by the relations established between the functions performed by the components of an organism, but also each function is explained by its contribution to the closure to efficient cause. As in the case of natural selection, also in relational biology the explanandum to which the theory wants to respond is part of the explanans.

To finish this section, it is relevant to note the similarity between the concept of closure, which defines the organism in relational biology, and that of the organism's form in Aristotle's teleology, which explains the existence of an internal agency that maintains the purposes of the organism:

The end is "internal" to the organism because it is the organism's form. And that form is a cause since, "Except for the organism's form (...) none of the parts that contribute to the organism's life would come to be or exist'. Those parts come to be, or are retained, because they support that form, and the form is therefore a cause qua end (Lenox as quoted in Short (2002, 325-326)).

\section{Piece's Signs and Final Cause}

According to Short (2002, 2007), Peirce takes Aristotle's explanation of the final cause as opposed to the efficient cause, and argues the need to introduce the former into modern science:

(...) we must understand by final causation that mode of bringing facts about according to which a general description of result is made to come about, quite irrespective of any compulsion for it to come about in this or that particular way (...) The general result may be brought about at one time in one way, and at another time in another way (...) Efficient causation, on the other hand, is a compulsion determined by a particular condition of things, and is a compulsion acting to make that situation begin to change in a perfectly determinate way; and what the general character of the result may be in no way concerns efficient causation (Peirce, as quoted in Short $(2007,136))$.

\footnotetext{
8 The union of a solid and a hollow arrowhead represents the fact that the outputs of the components can be efficient and material causes of other components in the organism.
} 


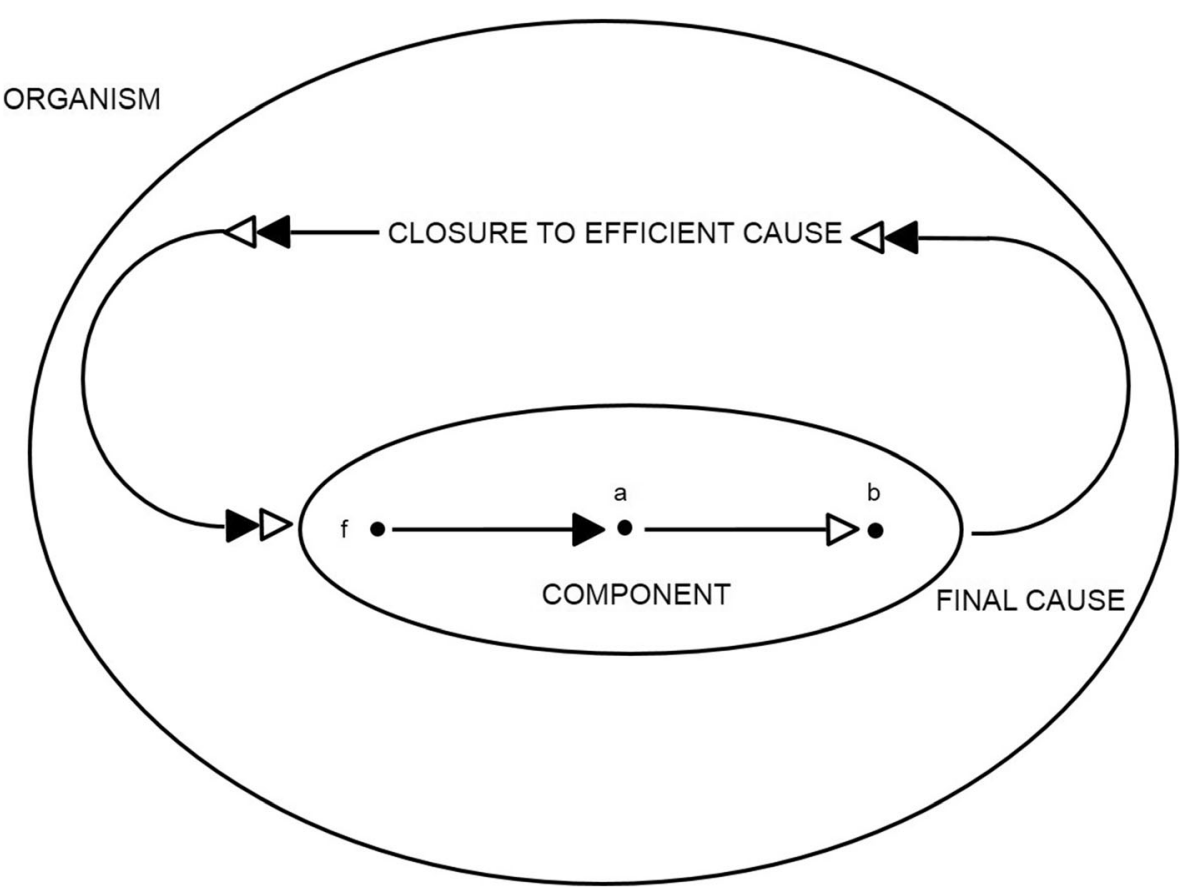

Fig. 7 Representation of the final cause

Peirce was inspired by statistical mechanics and natural selection, which, in contrast to Newton's mechanics, could not be explained by the natural science concept of cause. In these disciplines, mechanistic explanations were no longer sufficient; a teleological explanation was also needed. Peirce realized that Darwin had introduced a new explanatory principle, natural selection, according to which characteristics are selected for the types of effects that lead to reproductive success. These characteristics are selected for a purpose, even if they are sometimes unable to fulfill that purpose. Natural selection had reintroduced purposes, Aristotle's final cause, into modern science.

Peirce's semiosis is a logical and scientific study of signs action in nature and, to develop a theory of logic and to study nature, a type of actions that cannot be characterized as ordinary physical actions must also be considered, semiotic actions:

It is important to understand what I mean by semiosis. All dynamical action, or action of brute force, physical or psychical, either takes place between two subjects (whether they react equally upon each other, or one is agent and the other patient, entirely or partially) or at any rate is a resultant of such actions between pairs. But by "semiosis" I mean, on the contrary, an action, or influence, which is, or involves, a cooperation of three subjects, such as a sign, its object, and its interpretant, this tri-relative influence not being in any way resolvable into actions between pairs (...) and my definition confers on anything that so acts the title of a "sign." (Peirce 1931-1936, vol. 5, p. 484). 
Semiotic actions constitute the explanandum posed by Peirce's theory of signs. As in the cases of natural selection and relational biology, it is an explanandum that cannot be explained with the laws of physics alone; an explanandum that requires a new explanans. And in this case too, the explanandum is part of the explanans.

The concepts of sign, object and interpretant are inseparable from the triadic relation established between them. These three "subjects" are not a sign, an object and an interpretant by themselves, but by the role that each one of them plays in the semiotic relation. The semiotic action is explained by the realization of the interpretant, the sign and the object as subjects of the semiotic relation, but on the other hand, it is the semiotic action that defines the interpretant and the sign and the object as such, and the triadic relation. Peirce's sign is an explanandum that figures in the explanans and his semiosis rests on the concept of final cause.

Returning to the comparative analysis of the theories of Peirce and Rosen, it is appropriate to analyze the final cause in Peirce's semiosis relying on the representation of a semiotic relation with two mappings and following a method similar to that used when studying the final cause of a component of a relational model. The representation of the semiotic relation that has been proposed shows that the analysis of the final cause of a semiotic action can be broken down into two questions that correspond to the realization of the interpretant and the interpretation of the sign. Why the interpretant? Because it leads the agent from the sign to the object. Why the object? Because it determines that the sign determines the interpretant. Unlike the final cause of a component of an organism in relational biology, whose answer only requires the closure to efficient cause of the system, the explanation of the final cause of a semiotic relation also requires a local closure between the two constituents of the semiotic relation: the definition of the sign and its interpretation.

\section{Signs in an Organism}

The formal similarities and differences established in the previous sections between the theories of Rosen and Peirce, as well as the analysis of the role that the final cause plays in them constitute an adequate tool to discuss the existence of signs that realize and are interpreted inside organisms, including the simplest. It is convenient to start with the concept of interpretation.

\section{Short's Account of Interpretation}

Short's (2007) definition of interpretation builds on the naturalized account of purpose, where a purpose is a general type of outcome or effect which explains the existence of something because it is selected for having effects of that general type. A purpose is a type of outcome that explains why there are outcomes of that type and it is explanatory because it is selected-for as a means.

Applying the concept of purpose to the sign defined by Peirce, Short's definition of interpretation is as follows: 
An interpretant interprets a representamen as a sign of an object if and only if (a) the interpretant is or is a feature of a response to the representamen for a purpose, (b) the interpretant is based on a relation, actual or past or apparent or supposed, of the representamen to the object or of things of the type of the representamen to things of the type of the object, and (c) obtaining the object has some positive bearing on the appropriateness of the interpretant to the purpose. (Short 2007, $158)^{9}$.

Two comments on the role played by the representamen, the object and the interpretant according to this definition. First, saying that a representamen is interpreted as a sign of an object does not imply that the presence of the representamen leads in all cases to obtaining the object. The object might not be obtained due to the fact that the interpretant is based on a relation of things of the type of the representamen to things of the type of the object that is either an actual but fallible relation, or a past or apparent or supposed relation.

Second, when "the interpretant interprets" is said, it should not be understood that the interpretant is the agent that performs the interpretation. The interpretant is just one of the three subjects that are part of the triadic relation that defines a semiotic relation, in which the object determines that the representamen determines the interpretant ("an effect upon a person").

And, Short points out:

A purpose has to be some agent's, that selects for that type of outcome; or it has to be some mean's, that is, something that is selected for its having results of that type. A purpose, in other words, must be possessed by something either as agent or means $(2007,135)$.

Neither the sign, nor the object, nor the interpretant act according to a purpose, and neither does the semiotic relation as a whole. The one who acts purposefully is the interpreter of the sign. Interpreters produce interpretants as a means to an end:

The sign's 'action' therefore depends on its relevance to the purposes of an agent; only so does it have an effect. The sign makes or can make a difference: in that sense it 'acts', when it acts at all. But it acts only through influencing an agent that, independently of that sign, is pursuing some purpose. Talk of a sign's action is only another way of talking about how a sign determines its interpretant. Nothing is a sign except for its objective relevance to the purposes of possible agents (Short, 2007, 172).

In Peirce's semiosis, the purpose is not of the sign, nor of the object, nor of the interpretant; the purpose is of the interpreter.

\footnotetext{
9 In Short's original, interpretant, representamen, object, and purpose are represented by the letters R, X, O, and P. To facilitate explanation, in this quotation the letters have been replaced by the concepts they stand for.
} 


\section{Intentionality}

On the other hand, Short (2007) argues that, as the concepts of sign, interpretation and meaning are intentional concepts, in which the objects that are assigned to the signs do not need to exist to be assigned, and the intentional action is limited to animals, then only in the animal realm can one speak of sign, interpretation and meaning.

Conversely, the application of the concept of semiotic action to the analysis of the simplest organism is advocated below, based on what has been stated about relational biology, Peirce's semiosis and Short's definition of interpretation. The concept of sign is naturalized, analyzing the requirements that must be fulfilled to affirm the existence of signs in an organism. Previously, it is convenient to make a brief comment on the concept of intentionality, as it is understood in biosemiotics.

Favareau and Gare (2017) conducted a survey among experts in biosemiotics from different fields of study, in which respondents were presented with nine selected quotations and asked to report how suitable or unsuitable to the biosemiotic analysis they found the conceptualizations of "intentionality" reflected in each quotation.

The quotations that got the most approval from respondents were Hoffmeyer and Stjernfelt. It is interesting to copy the later:

It is the cyclical organization of metabolism which makes it meaningful to speak of 'intention' (whether conscious or not), because the directedness of intention, be it inside the organism or directed outwards into the niche is governed by the cyclical attractor of metabolism. ... Thus the biosemiotics vocabulary centered, like Kant predicted and Cassirer further argued, around the concept of intentionality, of telos, formally interpreted as cyclic pattern or order, gives meaning in relation to the notion of the cyclical flow of metabolism. (Stjernfelt as quoted in Favareau and Gare (2017, pp. 227-228)).

In accordance with what is stated in this paper, to properly grasp the concept of intentionality, "the cyclical organization of metabolism" should be replaced by "the closure to efficient cause of the system", which explains the self-maintenance, the self-repair and the replication of the system.

\section{Components and Signs}

According to relational biology, an organism is a material system closed to efficient cause and it can be analyzed as a set of components and the relations established between them. It is closure that gives the relations their own reality ${ }^{10}$, different from the properties of the components, and which differentiates living beings from inanimate material systems.

On the other hand, affirming the existence of a semiotic action in a cell is equivalent to affirming that both the definition of a semiotic relation (the determination of the interpretant) and its interpretation are realized within it. Furthermore, the three subjects

$\overline{10}$ According to Bateson (1972), the reality of ontological relations is exactly what distinguishes life from non-life. 
that constitute the triadic relation (the sign, the object and the interpretant), should be natural entities ${ }^{11}$. Consequently, a sign in an organism should necessarily be built on the components and relations that define it.

Suppose there are semiotic actions that are performed in an organism, such that signs are created and interpreted within it. In view of the above, the formal similarity that exists between the production of an output from an input in a component of an organism and the interpretation of a sign should be noted; such a similarity is specified in the correspondences between the interpretant and the efficient cause ${ }^{12}$, the representamen and the input, and the object and the output; a similarity by which the statement "the efficient cause produces the output from the input" would be equivalent to "the interpretant interprets the representamen as a sign of the object".

Thus, a relationship can be established between the concepts of component and semiotic relation in an organism and, with this, a definition of a semiotic relation can be proposed:

In an organism, a semiotic relation is the union of a component of the system and a relation between the entities that are part of that component, by which the output determines that the input determines the efficient cause. In this case, the efficient cause, the material cause and the output of the component correspond respectively to the interpretant, the sign and the object of the semiotic relation, and the action of the component corresponds to the interpretation of the sign.

Accordingly, the two constituents of a semiotic action, the formation of the semiotic relation and the interpretation of the sign are inseparable.

Moreover, it is convenient to distinguish between the two mappings with which a semiotic relation has been represented. First, as has been explained, the mapping in Fig. 5 corresponds to a component that, according to relational biology, contributes to the closure of the system to efficient cause, and has its efficient cause (the interpretant) generated inside the organism by the closure of the system. Furthermore, Fig. 5 represents the interpretation of the sign.

On the other hand, the mapping in Fig. 4 does not represent a component; it does not correspond to the production of the interpretant from the object and the sign. The interpretant is not the result of a component represented by this mapping (in fact this mapping does not represent any component). Rather, it is the result of some other component of the system that, with the production of the entity that will act as the interpretant, also contributes to the closure to the efficient cause of the organism. What the mapping in Fig. 4 represents is the determination of the interpretant, which is not the production of an entity that acts as such but its selection from among those produced by the closure of the system.

In conclusion, the realization of a semiotic action in an organism, the formation and interpretation of a sign, requires two types of closures; first, the closure to efficient cause that turns the material system into an organism, and to which the component that forms part of the semiotic relation contributes, and second, the closure established between the interpretation and the determination of the interpretant, which defines the semiotic relation.

\footnotetext{
11 The term "entities" refers to objects, processes, or a mixture of both.

12 As explained above, the correspondence must actually be established between the interpretant and the union of the efficient and formal causes.
} 
Consequently, an organism that included a sign could be represented as shown in Fig. 8.

\section{Purposes, Agents and Interpretation}

As previously argued, what makes a sign be a sign is its relevance to the purposes of an agent. The interpretant is a response to the sign for the purpose of an agent, where a purpose is a type of outcome that explains why there are outcomes of that type and it is explanatory because it is selected-for as a means. The one who acts purposefully is the interpreter of the sign.

Consequently, to account for the existence of signs in an organism, the question must now be posed as to which agent creates and interprets these signs.

As stated above, according to relational biology, (1) the final cause of the output of each component of an organism is its contribution to the closure to efficient cause and, with it, to the self-maintenance of the metabolism, self-repair and replication of the system; and (2) the realization of the closure to efficient cause is explained by the relations established between the functions performed by the components of an organism, but also each function is explained by its contribution to the closure to efficient cause.

Furthermore, it must be stated that if an organism includes a sign whose interpretation corresponds to the action of a component of the organism, then the purpose of the sign is the self-maintenance of the system, and the agent performing the

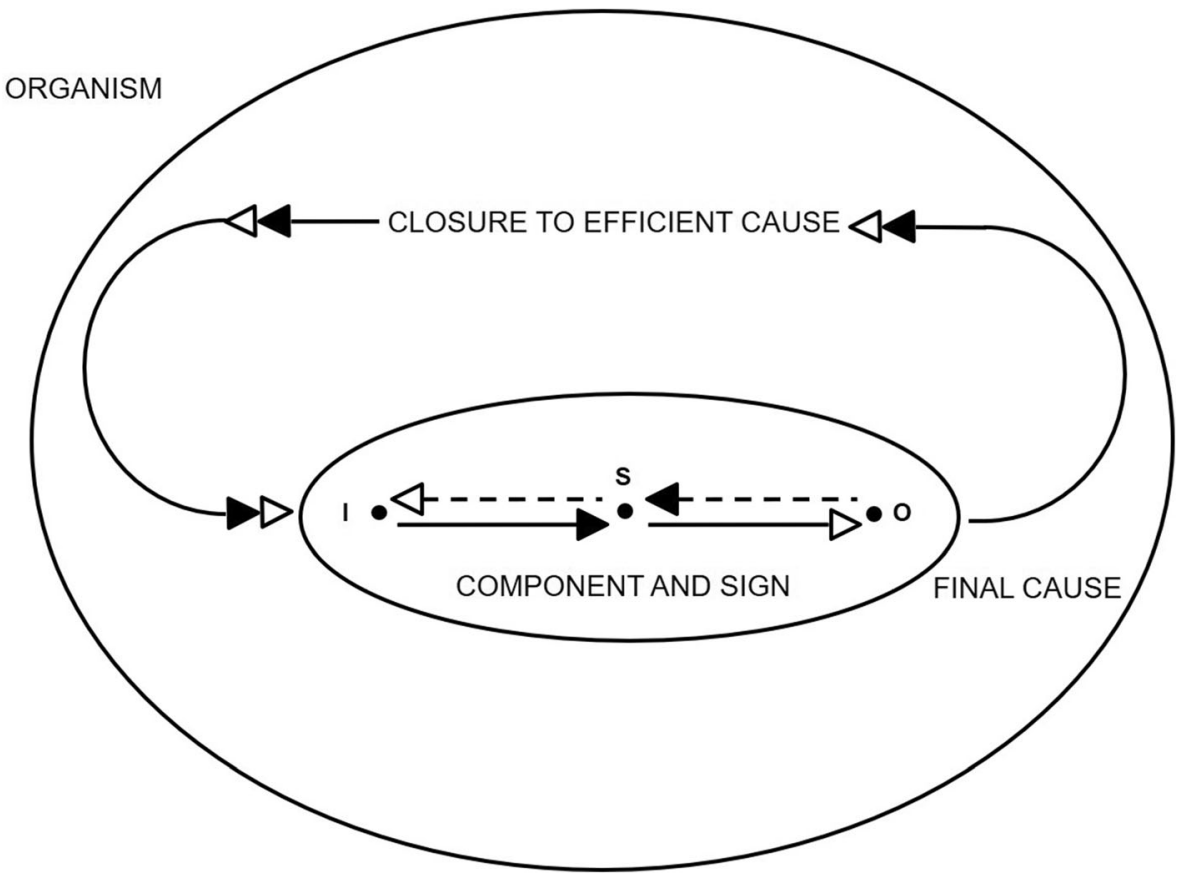

Fig. 8 An organism that includes a sign 
interpretation is the organism itself. Consequently, it can be stated that interpretation is a systemic process.

On the other hand, according to Hoffmeyer (2010, p.371), "The act of interpretation (...) seems to be a key to the production of meaning when this word is used in a situated local sense".

Two considerations are necessary to clarify this apparent contradiction. First, in an organism, the action of a component is locally performed but responds to the global self-maintenance of the system, i.e. to its closure to efficient cause.

Second, as stated, the existence of a sign in an organism implies the realization of two closures, a local one that defines the semiotic relation, and a global one, that defines the organism. Both closures constitute the necessary and sufficient condition for the realization of a sign in an organism. The action of a component corresponds to the interpretation of a sign only if the efficient cause is determined by the output of the component.

Accordingly, in an organism, the interpretation of a sign is locally realized but only because the systemic closure is realized. Thus, the interpretation of a sign in an organism is both a local and a systemic process.

Thus, the question of which agent performs each interpretation in an organism admits two complementary answers. A local one, which would correspond to the local production of meaning indicated by Hoffmeyer: the interpretation is realized by a part of the organism. But also, a global one: the interpretation is realized by the complete system defined by the closure: the organism. The interpretation of a sign, the production of a meaning, corresponds to the realization of a local biological function that contributes to the self-maintenance of the whole organism.

Furthermore, in an organism, it is necessary to distinguish different levels in which a hierarchy of closures and also a hierarchy of interpretations are articulated.

Consider, for example, the simplest multicellular organism. From the point of view of relational biology, in addition to the closure that defines each cell, the higher-level organism requires at least the realization of a closure to efficient cause in which its components are the cells that are part of it. It is the cells and the relations between them that define the multicellular organism.

On the other hand, there may be signs in the multicellular organism whose interpretation corresponds to the action of the cells, in which it should be true that a cell interprets an input it receives from outside as the sign of an output it produces, and the output that is produced determines (the state of) said cell.

\section{A Method of Analysis}

As a conclusion of the preceding sections, a method of analysis for the identification of signs in an organism is now defined. The goal pursued is to identify, if they exist, those biological processes that correspond to semiotic actions. The steps to pursue are the following.

First, to identify those processes that biology explains using concepts such as signals, codes or information, although it does not attribute any semiotic character to them. This is the case, for example, of the translation of messenger RNA chains into proteins or of the communication between distant cells. 
Second, to carry out a relational analysis, identifying the candidate process for semiotic action with the action of a component of the organism. To do this, the level of detail of the relational model to be used must be adequate for the biological process under study. The identification of the component implies the identification of its efficient cause, its input and its output, and also, due to the closure to efficient cause that defines the organism, its final cause, its function.

Third, to establish a correspondence of the entities identified as efficient cause, input and output with the concepts of interpretant, sign and object, and analyze if the object determines that the sign determines the interpretant. But, saying that the output entails that the input entails the efficient cause means not only that a specific input leads to a specific output (which occurs in all components), but that the efficient cause is also specific. If this is the case, the definition of a semiotic relation in an organism proposed above would be fulfilled and the presence of a sign could be affirmed whose formation responds to the determination of the interpretant (the efficient cause) and whose interpretation coincides with the action of the component and, therefore, with the realization of the analyzed process.

It should be noted that a sign identified by following these steps is a sign present in the organism (it is a sign for the organism), in which the entities identified as interpretant, sign and object form a triadic relation, and its interpretation conforms to Short's definition. In this case, it would be confirmed that the chosen process is really a semiotic action in which the concepts of sign, object, interpretant and interpretation (in its double aspect, local and systemic) have a precise definition, applicable to an organism.

Additionally, once a sign has been identified in an organism, it is worth asking what type of sign it is, according to Peirce's classification into icons, indices and symbols, a classification that is based on the type of relationship that exists between the sign and the object. The sign will be an icon if there are complementarities or correspondences between the sign and the object. It will be an index if it is not an icon, but the sign is actually affected by the object. Finally, it will be a symbol if it does not belong to either of the two previous types and its representative character consists precisely in its being a rule that determines the interpretant. An analysis of this relationship will certainly contribute to better understand the biological process being studied.

The proposed method combines the standard description of biological processes with an integrated account of Rosen's relational biology and Peirce's semiosis. In Vega (2021-II) it is applied to protein synthesis.

\section{Conclusions}

Life cannot be explained by the laws that apply to the inanimate world alone. Relational biology and biosemiotics propose theoretical frameworks to cover this gap. In this paper an integrated account of Rosen's Biology and Peirce's semiosis has been presented, highlighting the common points that can be found in these theories, but also the differences between them.

According to the analysis carried out, relational biology has been extended taking into account the relational character of the sign and the role of semiotic actions in the closure to efficient cause. 
Furthermore, the semiotic explanation of the organism has been based on the naturalization of the concepts of sign and interpretation, which have been built upon Rosen's biology. Short's definition of interpretation has also been an important tool for this task.

Three specific conclusions can be summarized as follows. First, in an organism, a semiotic relation is the union of a component of the system and a relation between the entities that are part of it, by which the output determines that the input determines the efficient cause. In this case, the efficient cause, the material cause and the output of the component correspond respectively to the interpretant, the sign and the object of the semiotic relation, and the action of the component corresponds to the interpretation of the sign.

Second, the existence of a sign in an organism requires the realization of two closures, a local one that defines the semiotic relation, and a systemic one, that defines the organism. The action of a component corresponds to the interpretation of a sign only if the efficient cause is determined by the output of the component. The interpretation of a sign in an organism (defined above) is both a local and a systemic process: it takes place locally but only because the system closure to efficient cause occurs.

And third, the application of the concepts expounded, allows the definition of a method of identification of signs in an organism and its classification in icons, indices and symbols. In Vega (2021-II), the proposed concepts and the method of identifying signs in an organism are applied to the analysis of protein synthesis, integrating the descriptions of standard biology, relational biology and semiotics.

Funding Open Access funding provided thanks to the CRUE-CSIC agreement with Springer Nature.

Open Access This article is licensed under a Creative Commons Attribution 4.0 International License, which permits use, sharing, adaptation, distribution and reproduction in any medium or format, as long as you give appropriate credit to the original author(s) and the source, provide a link to the Creative Commons licence, and indicate if changes were made. The images or other third party material in this article are included in the article's Creative Commons licence, unless indicated otherwise in a credit line to the material. If material is not included in the article's Creative Commons licence and your intended use is not permitted by statutory regulation or exceeds the permitted use, you will need to obtain permission directly from the copyright holder. To view a copy of this licence, visit http://creativecommons.org/licenses/by/4.0/.

\section{References}

Bateson, G. (1972). Steps to an ecology of mind. Ballantine Books

Favareau, D. (2009). The Logic of Signs. In: Essential Readings in Biosemiotics. Biosemiotics (Vol. 3). Springer

Favareau, D., \& Gare, A. (2017). The biosemiotic glossary project: Intentionality. biosemiotics 10, 413-459. https://doi.org/10.1007/s12304-017-9309-4

Hoffmeyer, J. (2008). Semiotic scaffolding of living systems. In Barbieri, M. (Ed.), Introduction to biosemiotics. Springer. https://doi.org/10.1007/1-4020-4814-9_6

Hoffmeyer, J. (2010). A biosemiotic approach to the question of meaning. Zygon (r), 45(2), 367-390. https:// doi.org/10.1111/j.1467-9744.2010.01087.x

Hoffmeyer, J. (2013). Why do we need a semiotic understanding of life? In Henning, B. G. and Scarfe, A. (Eds.) Beyond Mechanism: Putting Life Back Into Biology. Lexinton Books

Louie, A. H. (2009). More than life itself: A synthetic continuation in relational biology. Ontos Verlag 
Maturana, H., \& Varela, F. J. (1973). De máquinas y seres vivos: una teoría sobre la organización biológica. Editorial Universitaria S. A

Peirce, C. S. (1931-1936). The collected papers, Vol. 1-6. In: Hartshorne, C., Weiss, P. (Eds). Harvard University Press

Peirce, C. S. (1998). The essential Peirce, Vol. 2. Peirce Edition Project. Indiana University Press

Piaget, J. (1967). Biologie et Connaissance. Gallimard

Rosen, R. (1991). Life itself. A comprehensive enquiry into the nature, origin and fabrication of life. Columbia University Press

Rosen, R. (2012). Anticipatory systems. Philosophical, mathematical, and methodological foundations, 2nd Edn. Springer. (1st edn, 1985, Pergamon Press)

Short, T. L. (2002). Darwin's concept of final cause: neither new nor trivial. Biology and Philosophy, 17(3), 323-340

Short, T. L. (2007). Peirce's theory of signs. Cambridge University Press

Varela, F. J. (1979). Principles of biological autonomy. Elsevier

Vega, F. (2018). A critique of Barbieri's code biology through Rosen's relational biology: Reconciling Barbieri's biosemiotics with Peircean biosemiotics. Biological Theory 13, 261-279 (2018). https://doi. org/10.1007/s13752-018-0302-1

Vega, F. (2021-II). An integrated account of Rosen's relational biology and Peirce's semiosis. Part II: analysis of protein synthesis. Biosemiotics. https://doi.org/10.1007/s12304-021-09438-8

Publisher's Note Springer Nature remains neutral with regard to jurisdictional claims in published maps and institutional affiliations. 